both a priori and experimental evidence that such an insertion must stretch the molecule and give rise to some degree of unwinding of the helix. When the dye is added to the circular DNA, the superhelix untwists, in order that the number of turns in the primary helix may remain invariant. With addition of further dye, a superhelix of the opposite sense begins to form, so as to take up more slack, until a rather rigid structure is produced which cannot deform sufficiently to accommodate more dye. The circular form thus binds substantially less dye than the linear form. But when a dye is attached to DNA, there is a decrease in the density of the molecule; consequently the circular DNA saturated with ethidium has a gieater buoyant density than saturated linear DNA which contains more dye, and is widely separated from it by sedimentation in a caesium chloride density gradient. Excellent fractionations are achieved, which must exclude even molecules with a single break in one strand.

Electron microscopy on preparations from HeLa cells reveals, in addition to the circular molecules previously encountered in mitochondria (mean length 4.8 microns), some large supercoils which are multiples of these, and also some very small and polydisperse circles $(0 \cdot 2-3.5$ microns $)$. These could code only for between 200 and 3,500 amino-acids, and their function is unknown. It will be interesting to establish whether they are universal constituents of mammalian cells.

\section{Genome Transcription}

\section{from our Correspondent in Cell Biology}

In bacteria, unlike eucells, the replication of the DNA genome goes on throughout virtually the whole of the cell division cycle. This implies that transcription and replication of DNA occur simultaneously. Indeed, it has been suggested that the basal level of enzyme synthesis could be controlled by a coupling of these processes, with transcription occurring only at or near the replication point where stretches of single stranded DNA might be expected. This is not a universal mechanism, however, for, as Maaloe and Kjelgaard (1966) showed, ribosomal, transfer and some species of messenger RNA are made throughout the whole cell cycle.

Several important features of the pattern of DNA transcription and replication during the division cycle of Escherichia coli have recently been determined by Cutler and Evans (J. Mol. Biol., 26, 91; 1967) using an ingenious technique they devised (ibid., p. 81) for the isolation of segments of the $E$. coli genome. Synchronously dividing cultures of $E$. coli were fed pulses of 5-bromouracil at different times throughout the division cycle. If the population is sufficiently well synchronized, all cells will have the same region of DNA labelled during any one pulse. The labelled cells are then collected, their DNA extracted and sedimented in a density gradient which separates the denser 5-bromouracil labelled DNA from the rest. The various DNA fractions labelled at different times in the cell cycle fail to hybridize with each other to any great extent; thus they appear to be unique segments of the genome.

Cutler and Evans have been able to measure the amount of RNA which is transcribed off each of these segments at particular times in the cell cycle by the determination-using a $\mathrm{P}^{32}$ pulse labelling technique- of the amount of RNA synthesized during the cell cycle of a synchronous culture that will hybridize with each DNA segment and which is therefore homologous with it. They find that the entire genome is being transcribed continuously but that the levels of transcription of a particular segment show characteristic fluctuations during the cell cycle.

It is interesting that a high level of transcription of one region is usually accompanied by high levels for all the other segments. How this co-ordination of transcription of the whole genome is achieved remains to be seen. There is some evidence that control of ribosomal and/or transfer RNA transcription may be related to the position of the DNA replicating point, but this is not the case for most of the genome.

One most interesting observation is that two separate segments of the genome are complementary to $16 \mathrm{~S}$ and $23 S$ ribosomal RNA and most species of transfer RNA. This may mean there are two distinct sites containing the genes for these RNA species although the inherent limitations of hybridization experiments make this interpretation equivocal.

\section{Can Algae utilize Methane? from our Correspondent in Microbiology}

Reviewing hydrocarbon fermentations and their industrial application, A. E. Humphrey (Biotech. Bioeng., IX, 3; 1967) writes, "Until recently, the primary industrial concern with hydrocarbon microbiology was in connexion with oil prospecting, corrosion problems and formation of a microbial sludge in jet fuel tanks". The scope of hydrocarbon microbiology has expanded rapidly in the meantime and currently the quest is for means of exploiting this microbial activity commercially. Propitious reports of the use of microbes in the partial refining of crude oil fractions and in the production of edible yeast and several biochemicals from hydrocarbons provide a great stimulus for continued research and development.

A report from Dr Lennart Enebo working at the Royal Institute of Technology in Stockholm (Acta Chem. Scand., 21, 625; 1967) may charge the interest in these matters still further. While studying the growth of photosynthetic sulphur bacteria in different gaseous environments Dr Enebo isolated the green alga Chlorella from highly reducing enrichment media in which carbonate and methane provided the carbon sources. Passage of the alga on a glucose medium led to an almost complete, but not total, elimination of the associated microflora. The addition of high quality methane to the carbonate medium culminated in a 35-45 per cent enhancement of algal growth. The grounds for believing that the alga assimilates the hydrocarbon hinge largely on two points. First, the alga excretes various metabolites which are likely to inhibit the growth of methane oxidizing bacteria. Second, free oxygen is produced by algal cultures growing in an atmosphere of 100 per cent methane and it seems reasonable to suppose that this may act in the oxidation of the paraffin to methanol. The subsequent assimilation of $\mathrm{C}_{1}$ units as carbon dioxide or their incorporation into sugar phosphates has been postulated but not proved. Enebo's claim for methane utilization by Chlorella is supported by the fact that he was unsuccessful in demonstrating other methane 